\title{
The required aerodynamic simulation fidelity to usefully support a gas turbine digital twin for manufacturing
}

\section{Original article}

Article history:

Submission date: 2 October 2020

Acceptance date: 29 December 2020

Publication date: 18 February 2021

This is the updated version of a paper originally presented at the Global Power and Propulsion Virtual Technical

Conference, GPPS Chania 20, Sept. 7-9, 2020.

\section{Check for updates}

*Correspondence:

WYL: leewenyao89@gmail.com

\section{Peer review:}

Single blind

\section{Copyright:}

(c) 2021 Lee et al. () This is an open access article distributed under the Creative Commons Attribution Non Commercial No Derivatives License (CC BY-NC-ND 4.0). Unrestricted use, distribution, and reproduction of the original work are permitted for noncommercial purposes only, provided it is properly cited and its authors credited. No derivative of this work may be distributed.

\section{Keywords:}

manufacturing variability; real geometry; Digital Twin; computational fluid dynamics

\section{Citation:}

Lee W. Y., Dawes W. N., and Coull J. D. (2021). The required aerodynamic simulation fidelity to usefully support a gas turbine digital twin for manufacturing Journal of the Global Power and Propulsion Society. 5: 15-27. https://doi.org/10.33737/jgpps/132007

\author{
Wen Yao Lee ${ }^{1, *}$, William N. Dawes ${ }^{1}$, John D. Coull ${ }^{2}$ \\ ${ }^{1}$ Whittle Laboratory, University of Cambridge, Cambridge, United Kingdom \\ ${ }^{2}$ Oxford Thermofluids Institute, University of Oxford, Oxford, United Kingdom
}

\begin{abstract}
With the imminent digitalisation of the manufacturing processes of gas turbine components, a large volume of geometric data of as-manufactured parts is being generated. This geometric data can be used in aerodynamic simulations to predict component performance. Both the cost and accuracy of these simulations increase with their fidelity. To efficiently exploit Digital Twin technology, one must therefore understand how realistic the aerodynamic simulations need to be to give useful performance predictions.

This paper considers this issue for a sample of scrapped high-pressure turbine rotor blades from a civil aero engine. The measured geometric data was used to build aerodynamic models of varying degrees of realism, ranging from quasi-three-dimensional blade sections for an Euler solver to three-dimensional, multi-passage and multi-stage Reynolds-AveragedNavier-Stokes models. The flow near the tip of these shrouded blades is sensitive to manufacturing variability and can switch between two quasistable horseshoe vortex modes. In general, capacity and exit flow angle can be adequately predicted by three-dimensional, single-passage calculations: averaging single-passage calculations gives a good prediction of the multi-passage behaviour. For efficiency and stage loading, the approach of averaging single-passage calculations is less accurate as the multipassage behaviour requires an accurate prediction of the horseshoe vortex modes.
\end{abstract}

\section{Introduction}

Digital Twin technology is generally considered to be part of the wider Industry 4.0 revolution, whereby the digitalisation of various industries opens up new opportunities to exploit data to improve products and processes.

\section{Digital twin}

The term "Digital Twin" is widely used and has a range of accepted definitions. The term generally refers to an integration of data between physical and virtual components (Fuller et al., 2020). In the current context of the manufacturing process of gas turbine blades, a Digital Twin incorporates structured-light scans and aerodynamic performance (primary functional requirement) predictions. The scan data traces the unique geometry of each component and the aerodynamic predictions can be used to sentence components and compensate for manufacturing variability (Lee et al., 2019a). 


\section{Aerodynamic simulation hierarchy}

The aerodynamic environment of a turbine blade is complex and can only be captured using physics-based simulations. Computational Fluid Dynamics (CFD) tools that encompass a range of fidelities have been used by various published studies and they will be discussed here in the order of increasing fidelity.

Considering the high volume of manufactured blades, rapid CFD tools like the Multiple blade Interacting Streamtube Euler Solver (MISES) (Drela, 1985) are attractive. MISES is a streamtube Euler solver coupled to an integral boundary layer code that is limited to quasi-three-dimensional (Q3D) blade sections. It has been used to assess compressor blades (Garzon and Darmofal, 2003; Dow and Wang, 2015; Goodhand et al., 2015) and turbine blades (Duffner, 2008).

Reynolds-Averaged Navier-Stokes (RANS) CFD solves the time-averaged Navier-Stokes equation. By incorporating source terms, turbulence models, and mixing planes, full three-dimensional (3D) geometries of multipassage and multi-blade-row turbomachinery can be studied. However, RANS CFD is not able to resolve timedependent phenomena and is vulnerable to turbulence and transition modelling. With today's computational resources, RANS CFD is the favoured tool for predicting the aerodynamics performance of manufactured gas turbine blades (Lange et al., 2012; Högner et al., 2016).

Unsteady RANS (URANS) CFD allows time-accurate fluctuations in the flow field to be resolved. URANS can capture large-scale movements in the fluid but does not resolve the vortical structures in the boundary layer. In addition, the computational cost of URANS models often make them impractical for the purposes of design, let alone for high-volume assessments. If resources permit, URANS CFD could provide useful insights for dynamic blade loading (Clark et al., 2018).

The highest degrees of fidelity are offered by Large Eddy Simulations (LES) and Direct Numerical Simulation (DNS). These tools can resolve flow features at the length scales of surface roughness (Kapsis and He, 2018). However, given their immense computational cost, these are not currently practical options for Digital Twins, and are therefore not considered in this paper.

Increasing the fidelity of the CFD methods improves the realism and accuracy of the predictions but it is also associated with higher computational cost and complexity. Given the high volume of parts that need to be assessed, a balance must be struck between computational expense and accuracy. This paper therefore examines the question: what is the trade-off between cost and accuracy of different aerodynamic simulation fidelities?

\section{Paper aims \& outline}

This paper compares the accuracy and computational cost of a range of aerodynamic simulation methods. Using the geometric data from a set manufactured turbine blades, the predictions of key aerodynamic performance parameters are compared across the range of simulation fidelity. The following sections detail:

- the aerodynamic simulation methods;

- the geometry measurement and processing methods, and;

- the results obtained from the various simulations, highlighting differences in capability and cost.

The paper concludes with recommendations on the suitable aerodynamic simulations to support Digital Twins.

\section{Methodology I - aerodynamic simulation methods}

The aerodynamic simulation methods that are considered in this paper are shown in Figure 1: Euler models, unshrouded and shrouded single-passage-single-stage RANS models, multi-passage-single-stage RANS models, single-passage-multi-stage RANS models, and single-stage URANS models. MISES was used as the Euler solver and Rolls-Royce's in-house CFD solver, Hydra (Moinier and Giles, 1998), was used for the RANS and URANS models.

The approach taken for this study is purely computational. It would also be possible to experimentally test parts with manufacturing variability and compare the results to the predictions from simulations. However, many of the performance changes observed are within the accuracy range of even very well-conditioned experiments, especially when compressibility effects are considered. The paper therefore ultimately references the simulations to the highest fidelity case (the URANS models). 


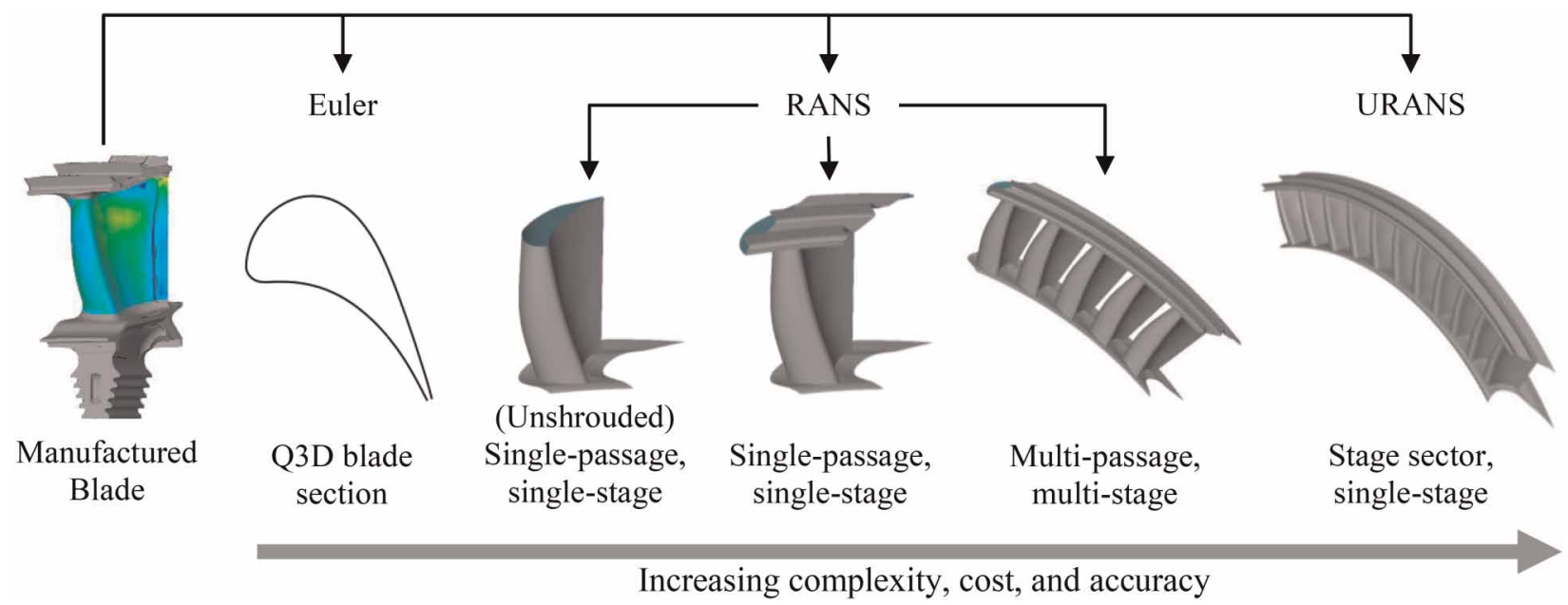

Figure 1. Hierarchy of aerodynamic simulation methods.

\section{Euler solver: MISES}

Version 2.56 of MISES was used and the models were run to the prescribed pressure ratio. The models were run for 10 inviscid iterations before introducing viscous effects for a further 15 iterations. This made the model more robust by allowing the flow field to stabilise before introducing the boundary layers (Drela and Youngren, 2008).

\section{RANS solver: Hydra}

Hydra's spatial discretisation is based on an upwind edge-based finite volume scheme and is second-order accurate. Hydra has been experimentally validated for a similar geometry (Zamboni and Adami, 2016) and across a range of turbine designs (Coull, 2017). Rolls-Royce's Parametric Design \& Rapid Meshing (PADRAM) (Shahpar and Lapworth, 2003) was used to generate multi-block, structured meshes. Unless otherwise specified, the computational domain is a 1-stage simulation of the High Pressure Turbine (HPT) stage, as illustrated in Figure 2. Sealing flows and film cooling are modelled using secondary inlets and source terms on the blade respectively.

The $k-\omega$ SST turbulence model (Menter, 1992) with wall functions was used favouring its robustness across the range of freestream and highly viscous flow regimes typically found in a HPT. The PADRAM mesh has wall $y^{+}$values of approximately 30 or higher on the blades and endwalls, in line with the minimum $y^{+}$requirements of the wall functions. Mesh sensitivity studies on a similar case have been previously published (Lee et al., 2019a) which showed residual mesh sensitivity of $\sim 0.01 \%$ for the HPT stage inlet capacity, $Q$ and $\sim 0.5$ in scaled HPT stage efficiency, $\eta$. These residuals are a small fraction of the observed range of performance variations (see Figure 10).

This approach contains approximations, including: the inability of RANS models to resolve time-dependent phenomena (e.g. TE shedding), film cooling being represented by source terms, and the turbulence model. Broadly speaking, these uncertainties behave systemically and assuming a uniformly distributed impact on the results, the effect of these approximations on the predicted performance changes from the design-intent is significantly reduced.

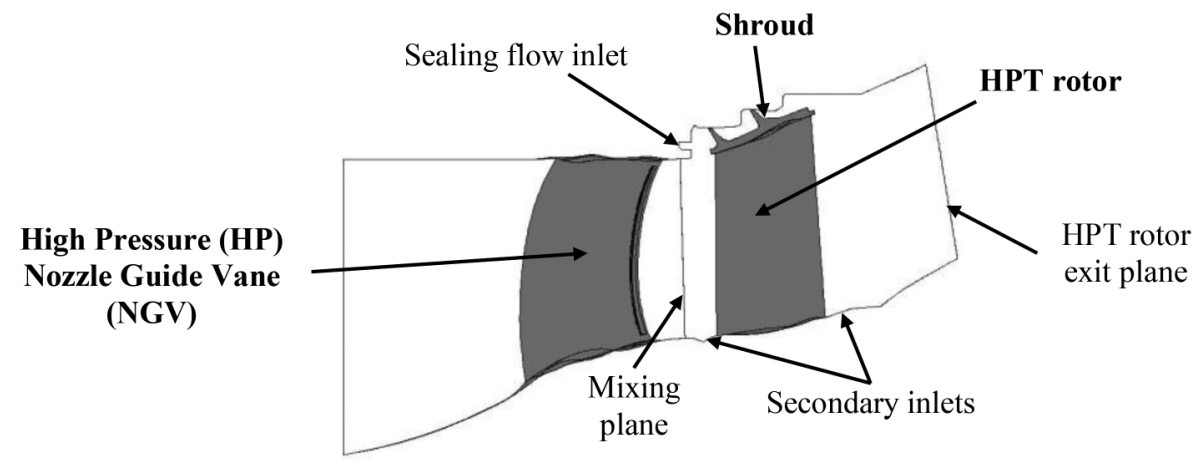

Figure 2. Meridional view of the 1-stage CFD model. 


\section{Key performance parameters}

For a turbine stage, the performance quantities of interest are: the exit flow angle, $\beta_{\text {exit }}$, the stage inlet capacity, $Q$, the stage efficiency, $\eta$, and the stage loading coefficient, $\psi \cdot \beta_{\text {exit }}$ is defined in Equation (1) and computed from the mass-averaged tangential velocity, $V_{\vartheta}$, and the mass-averaged axial velocity, $V_{x}$, at the rotor exit plane. The $\beta_{\text {exit }}$ of the rotor is particularly important and it controls the produced work, the passage mass flow, and the stage reaction.

$$
\beta_{\text {exit }}=\operatorname{atan} \frac{V_{\vartheta}}{V_{x}}
$$

$Q$ is defined in Equation (2) as a quasi-non-dimensional where $\dot{m}_{\text {inlet }}$ is the mass flow at the stage inlet, and $T_{0, \text { inlet }}$ and $P_{0 \text {,inlet }}$ are the mass-averaged stagnation temperature and stagnation pressure at the stage inlet.

$$
Q=\frac{\dot{m}_{\text {inlet }} \sqrt{T_{0, \text { inlet }}}}{P_{0, \text { inlet }}}
$$

For the purposes of this study, $\eta$ is defined in Equation (3) and obtained by calculating the rotor torque by numerically integrating forces over all the specified rotating wall faces (surface, $S$ ) and summing the pressure, $p$, and viscous shear, $\tau$, terms (Rolls-Royce plc, 2009).

$$
\eta=\frac{\sum_{i=\text { rotating faces }}\left(\iint F_{\vartheta} r d S\right)_{i}+\sum_{i=\text { grid points }}(p)_{i}+\sum_{i=\text { grid points }}(\tau)_{i}}{\sum_{i=\text { inlets }}\left(\dot{m} h_{0}\right)_{i}-\left(\dot{m} h_{0, \text { ideal }}\right)_{\text {main exit }}}
$$

$\psi$ is defined in Equation (4) as the ratio of the change in total enthalpy, $h_{0}$, across the stage divided by the square of rotational speed of the rotor blade, $U$. The value of $U$ is area-averaged at the rotor exit plane.

$$
\psi=\frac{\Delta h_{0}}{U^{2}}=\frac{\sum_{i=\text { inlets }}\left(\dot{m} h_{0}\right)_{i}-\sum_{i=\text { exits }}\left(\dot{m} h_{0}\right)_{i}}{U^{2}}
$$

\section{Methods II - geometry measurement and recreation methods}

The ability to capture and recreate the geometry of manufactured blades in a numerical environment is essential for the Digital Twin. Suitable measurement methods include using a Coordinate Measuring Machine (CMM) (Garzon and Darmofal, 2003), structured-light scanning (Heinze et al., 2014), and computed tomography (Högner et al., 2017). Structured-light scanning is the method of choice as it produces tessellated point clouds, stereolithography (STL) meshes, with an accuracy of $8-27 \mu \mathrm{m}$ depending on the local curvature gradient (Kamenik et al., 2018). A sample of 25 HPT rotor blades was measured with an ATOS series scanner. The geometry is representative of a modern design and features a shroud, film-cooling holes, and thermal-barrier-coating. These blades, however, were scrapped and are therefore not representative of in-service parts. In this study, only the geometry changes in the HPT rotor blades' $3 \mathrm{D}$ aerofoil were considered. The effects of roughness were omitted as they would require higher fidelity simulation tools than RANS.

The geometric information from the STL meshes need to be extracted before it can be mapped to the relevant input files for MISES and PADRAM and this is shown in Figures 3 and 4. The STL meshes were first aligned using the fir tree (highlighted red in Figure 3) in order for the blades to be oriented as they would be in the engine. Since film cooling is modelled with source terms, a smooth blade surface is required and the film cooling holes were removed using BladeCleaner (Högner et al., 2015). Blade sections were then extracted at 21 circumferential stream surfaces. The as-scanned trailing edge (TE) steps cannot be modelled in MISES and they tend to introduce negative volumes in the PADRAM mesh. Thus, a circle was fitted in the vicinity of the TE and the TE step was omitted. The result is blade sections that preserve the geometry of the STL meshes, except in the region of the TE. This simplification is a source of uncertainty due to the limitation of using a structured meshing approach that is more suitable for parametrically defined blades.

Displacement fields were obtained by comparing the blade sections from the STL meshes to the design-intent. As the blades are manufactured "cold" and the engine runs "hot", the displacement fields were scaled to account 


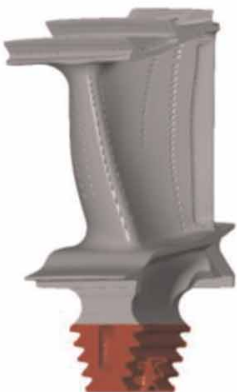

Alignment

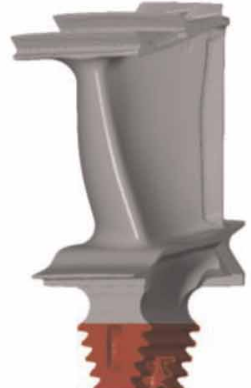

Remove Film Cooling Holes

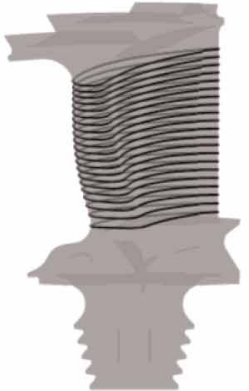

Extract Blade Sections
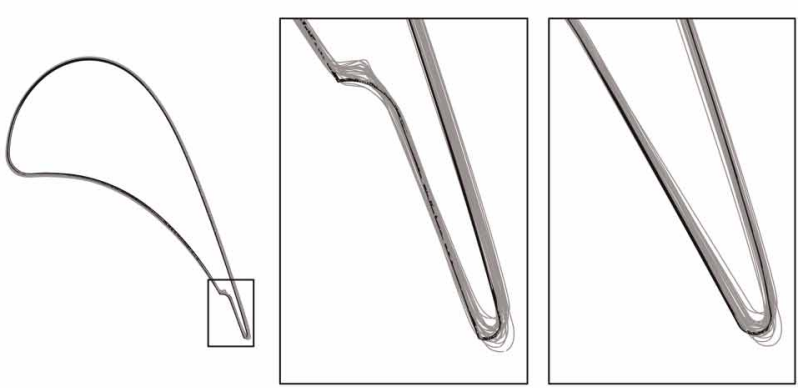

Simplify Blade Sections' Trailing Edges

Figure 3. The geometry recreation process including: STL mesh alignment, film cooling holes removal, blade sections extraction, and removal of the trailing edge step.

for thermal expansion by comparing the corresponding chord lengths between the physical and CFD blades. The scaled displacement fields were then mapped to the MISES and PADRAM blade sections, as shown in Figure 4 (Left). The span-wise geometry change is also well captured and this can be visualised by comparing the displacement fields from the STL mesh and the PADRAM reconstruction, as shown in Figure 4 (Right). Since MISES does not model the TE, the MISES blade sections were "cut-back" by $1 \%$ of the blade length. Finally, the coordinates of film cooling source terms were also perturbed to match the local displacements that were introduced by manufacturing variability.

\section{Results and discussion}

The results obtained from the various aerodynamic simulation methods will be discussed under the following topics, which compare the methods in Figure 1 in the order of increasing fidelity:

- Part I: Q3D Euler vs. Single-Passage 3D RANS

- Part II: Multi-Row and Multi-Stage Effects

- Part III: Multi-Passage Effects

- Part IV: Accuracy and Computational Costs across the Hierarchy

\section{Part I: Q3D Euler vs. single-passage 3D RANS}

The ability to accurately predict the $\beta_{\text {exit }}$ is vital as the bulk flow direction is one of the primary means by which the turbine's functional requirements on work, capacity, reaction, and efficiency are met. Figure 5 compares the trends of mass-averaged $\beta_{\text {exit }}$ that were obtained from the following models:
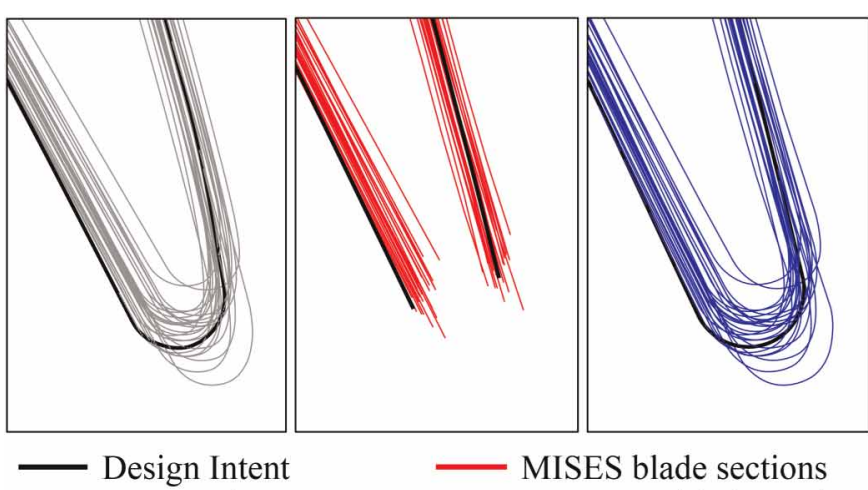

MISES blade sections

Measured Geometry

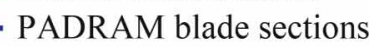

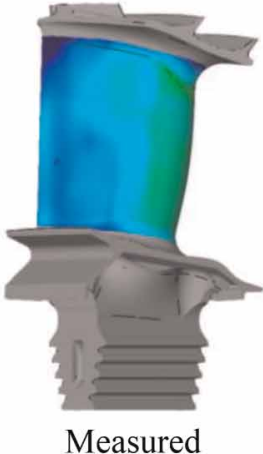

Measured Geometry

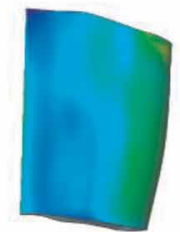
Reconstructed Geometry

Figure 4. (Left) Mapping the measured blade geometry to the MISES and PADRAM blade sections. (Right) Displacement fields from an example STL mesh and its reconstruction in PADRAM. 


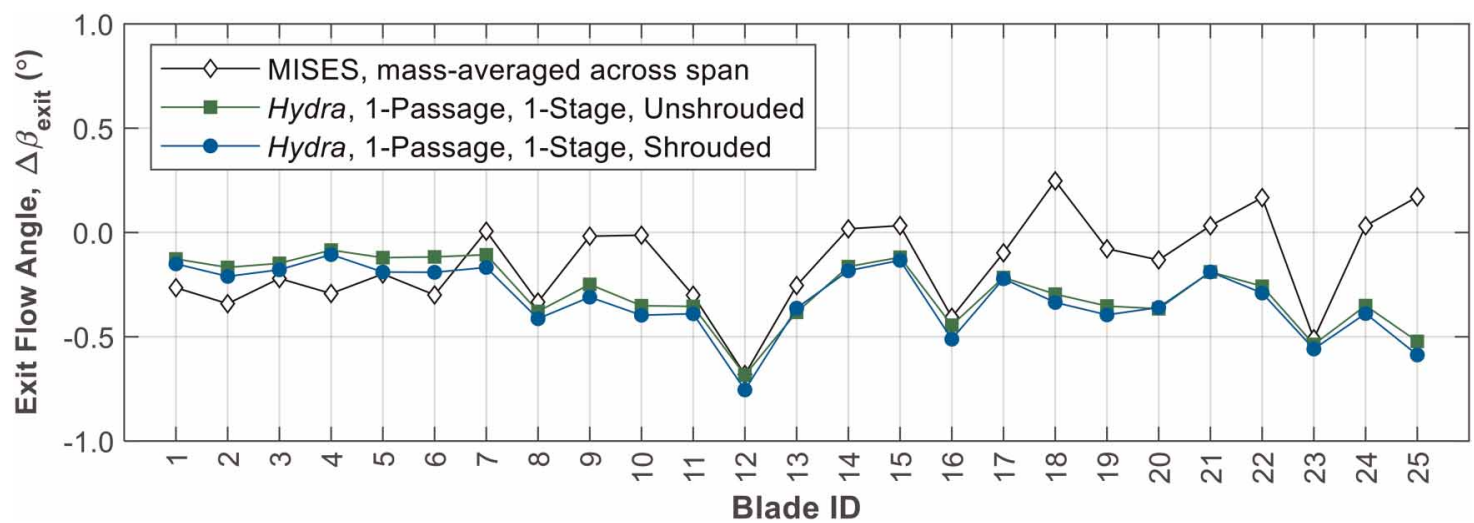

Figure 5. Predictions of mass-averaged $\beta_{\text {exit }}$ from the MISES and single-passage Hydra models.

- Q3D MISES models from the 21 blade sections extracted from each blade.

- 3D, unshrouded, 1-passage, 1-stage, Hydra RANS model.

- 3D, shrouded, 1-passage, 1-stage, Hydra RANS model.

The MISES results were mass-averaged across the span and the Hydra results were mass-averaged at the HPT rotor exit plane. Figure 5 shows that the trend from MISES does not match those of the 3D Hydra models completely and with notable discrepancies for blade IDs 18, 24, and 25. This is primarily due to MISES treating each Q3D blade section in isolation and not accounting for the effects of the hub and tip vortices. In addition, the trends from the unshrouded and shrouded models show that changes in the $\beta_{\text {exit }}$ is well-estimated even while omitting the shroud.

\section{Part II: multi-row and multi-stage effects}

For civil aero engines, the aerodynamic changes on one blade row will have a "knock-on" effect on all downstream blade rows. To study this, 3D RANS CFD models of an extended computational domain were created, incorporating the Intermediate Pressure (IP) NGV, Intermediate Pressure Turbine (IPT) rotor blade, and the first Low Pressure (LP) NGV. Figure 6 shows the radial profiles of the inlet and exit flow angles, referenced to the mass-averaged values across the respective inlet and exit planes, and gives a visual appreciation of the how small geometry changes in the HPT rotor blade row affects the flow angles of the entire IPT stage (IP NGV and IPT rotor blade).

Although the geometry changes were only imposed on the HPT rotor blade, Figure 6 shows that the downstream effects only diminish by the IPT rotor blade exit. Immediately downstream of the HPT rotor, the flow angle changes caused by the geometric variability imposed on the HPT rotor blade lowers the inlet flow angle to

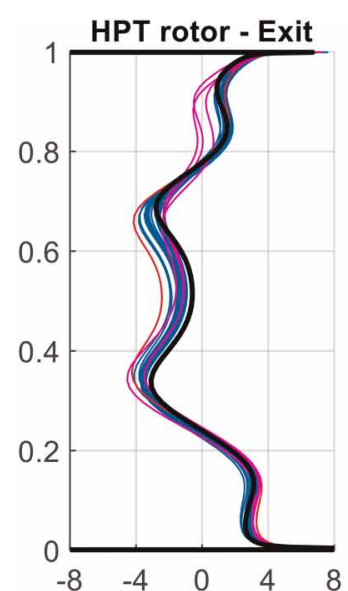

Rel. Flow Angle $\left({ }^{\circ}\right)$

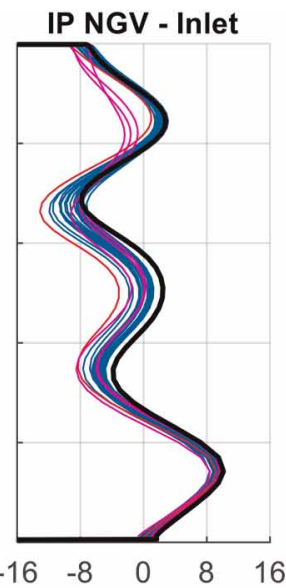

Abs. Flow Angle $\left({ }^{\circ}\right)$

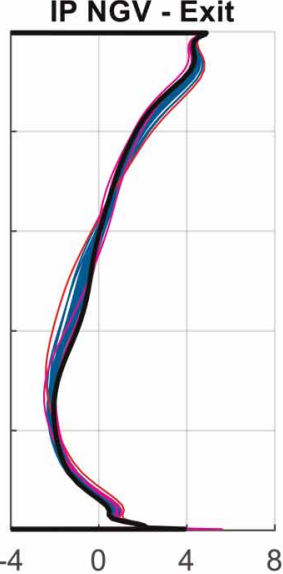

Abs. Flow Angle $\left({ }^{\circ}\right)$

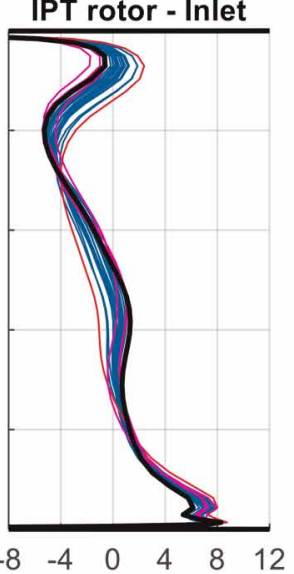

Rel. Flow Angle ( $\left.{ }^{\circ}\right)$

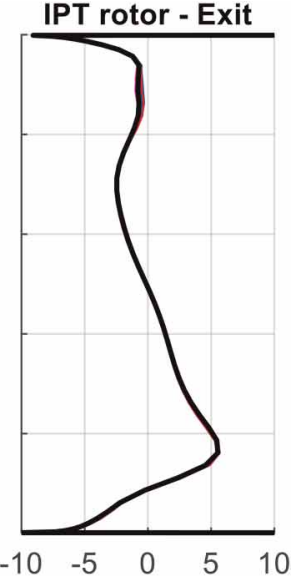

Rel. Flow Angle ( ${ }^{\circ}$ )

Figure 6. Radial profiles of flow angles from the HPT rotor exit and the IPT stage. "Abs." denotes "absolute" and "Rel." denotes "relative". Refer to Figure 8 for the corresponding efficiency plots. 

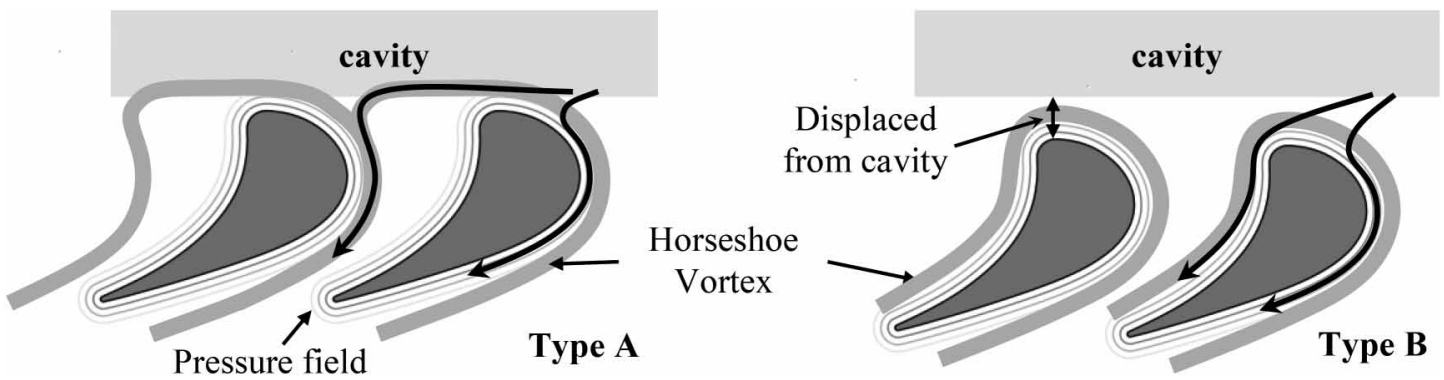

Figure 7. Illustration of the flow structures due to the blades' proximity to the cavity, showing (Left) the HSV partly in the cavity and (Right) the HSV outside the cavity.

the IP NGV (in the absolute frame) by up to $\sim 6^{\circ}$ around the mid-span. Although the IP NGV helps reduce the variability in the flow angle by the time the flow arrives at IP NGV exit plane, the IPT rotor's inlet flow angles are still being affected by up to $\sim 3^{\circ}$ in the mid-span (under-turning) and tip (over-turning) regions.

Examining the radial profile of $\beta_{\text {exit }}$ from the HPT rotor in Figure 6, three of the 25 blades show a distinct shift in $\beta_{\text {exit }}$ between $70 \%<r<95 \%$ (coloured magenta in Figure 6) and this is due to a change in the behaviour of the horseshoe vortex (HSV) (Lee et al., 2019b). The change in the HSV flow structure is illustrated in Figure 7. In "Type A" flow (design-intent) the HSV is largely held within the upstream cavity by the blades' pressure field. The legs of adjacent HSVs emerge together close to the suction-surface of the blade; thus the sealing flow remains close to the blade suction-surface.

If the influence of the blades' pressure field is weakened, either by a change in blade loading and/or a change in the position of the blade, the pressure side leg of the HSV emerges from the cavity and wraps around the blade leading edge. This alternative mode for the HSV is shown in Figure 7 as "Type B" flow. A more detailed discussion surrounding the mechanism behind this change in the flow field has been previously published (Lee et al., 2019b).

The changes in the flow angles in the IPT stage affects the efficiency of the stage and Figure 8 plots the changes in the scaled $\eta$ for the HPT stage (2.5-stage and 1-stage simulations) and the IPT stage. As shown, the IPT stage also responds to changes in the geometry of the HPT rotor blade. In Figure 8, two types of behaviour are highlighted and the corresponding profiles of $\beta_{\text {exit }}$ have also been highlighted in Figure 6:

- Red: Blade ID 12 exhibits Type A behaviour and both the HP and IP stage scaled $\eta$ decreases.

- Magenta: Blade IDs 13, 20, and 23 exhibit Type B behaviour but only the HP stage scaled $\eta$ decreases.

For blade ID 12, the HPT rotor exit flow angle was significantly reduced between $30 \%<r<70 \%$. This under-turning results in a lower work output and consequently, a lower HPT stage efficiency. This underturning cascades downstream to the IP NGV and redistributes the inlet flow angle profile to the IPT rotor blade. This shifts the work across the span towards the IPT rotor blade tip where losses are generally higher, reducing the IPT stage efficiency.

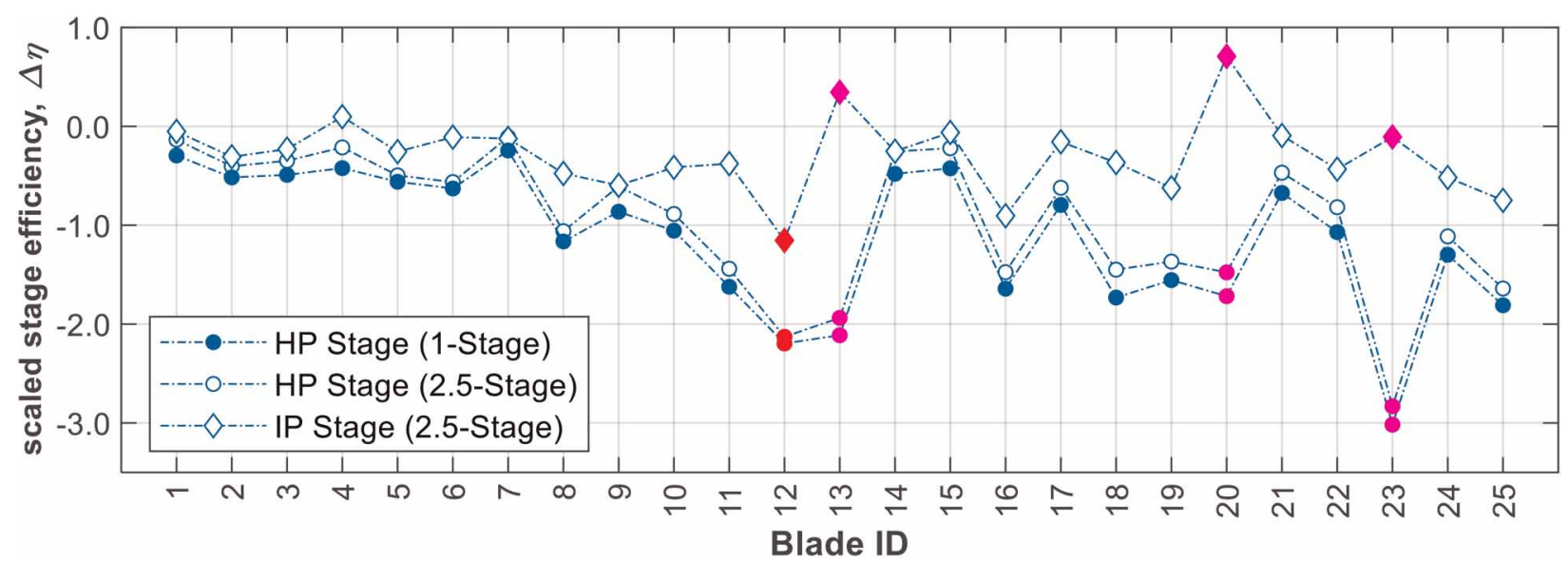

Figure 8. Scaled stage efficiencies obtained from the 1-stage and 2.5-stage Hydra RANS models. Refer to Figure 6 for the corresponding radial profiles of the flow angles. 
For blade IDs 13, 20 and 23, the change in HSV behaviour causes the tip region of the HPT rotor blade to under-turn the flow, producing less work and reducing HPT stage efficiency. However, unlike for blade ID 12, the IP NGV is effective is removing most of the flow angle discrepancy at the IP NGV inlet and the exit flow angle from the IP NGV is similar to the design-intent, minimising the impact of the change in HSV behaviour on the IPT stage efficiency.

The small differences in the trends between the 2.5-stage and 1-stage predictions of scaled $\eta$ of the HPT stage is due to a small change in the operating point of the turbine. Although the absolute numbers are slightly different, the trend of the change in HPT stage scaled $\eta$ is shared between the two types of simulations, as expected.

\section{Part III: multi-passage effects}

In an engine, adjacent blades will have slightly different manufacturing deviations. This interaction with neighbouring blades is not captured by single-passage calculations which assume periodicity over one blade pitch. Multi-passage simulations were therefore used to explore these interactions: 26 1-stage models each of 3-passage and 5-passage shrouded HPT rotor blades were created and solved using Hydra. The 26 cases include one case (case index 1) assembled using only blades that exhibit Type B behaviour, and a further 25 cases constructed using Latin Hypercube Sampling where geometric data from the 25 STL meshes were used to populate each blade position once.

As with the single-passage calculations, multi-passage cases also exhibited Type A and Type B flow. However, the flow structure for each multi-passage calculation does not simply depend on the presence of blade(s) that exhibited Type B behaviour in a single-passage calculation. Figure 9 compares:

- the flow structure observed in the multi-passage calculations (subscript MP) and

- the "average" of the flow structures that are observed in the single-passage calculations (subscript ASP) of the corresponding constituent blades.

Examining the trend for the 5-passage cases, Type B flow is observed in multi-passage cases 1, 3, 5, 6, 7, 9, 12, $15,17,18,23$, and 24 but cases $6,9,12$, and 24 do not contain blade(s) that gave Type B behaviour in a single-passage calculation. In addition, multi-passage cases 10, 14, 16, 21, 25, and 26 all contain a blade that gives Type B behaviour but all demonstrate Type A flow. Similar observations can be made for the 3-passage cases.

The above comparison shows that the detailed flow behaviour in the multi-passage environment is somewhat different to the single-passage environment. Nonetheless, if the overall performance can be estimated from an average of single-passage calculations, then the cost and complexity of simulations will be reduced enormously. Figure 10 shows this assessment for the four key performance parameters mentioned earlier:

- each subplot compares the multi-passage (MP) result with the average of single-passage (ASP) results;

- the accuracy of the averaging is quantified using an $R^{2}$ value; and

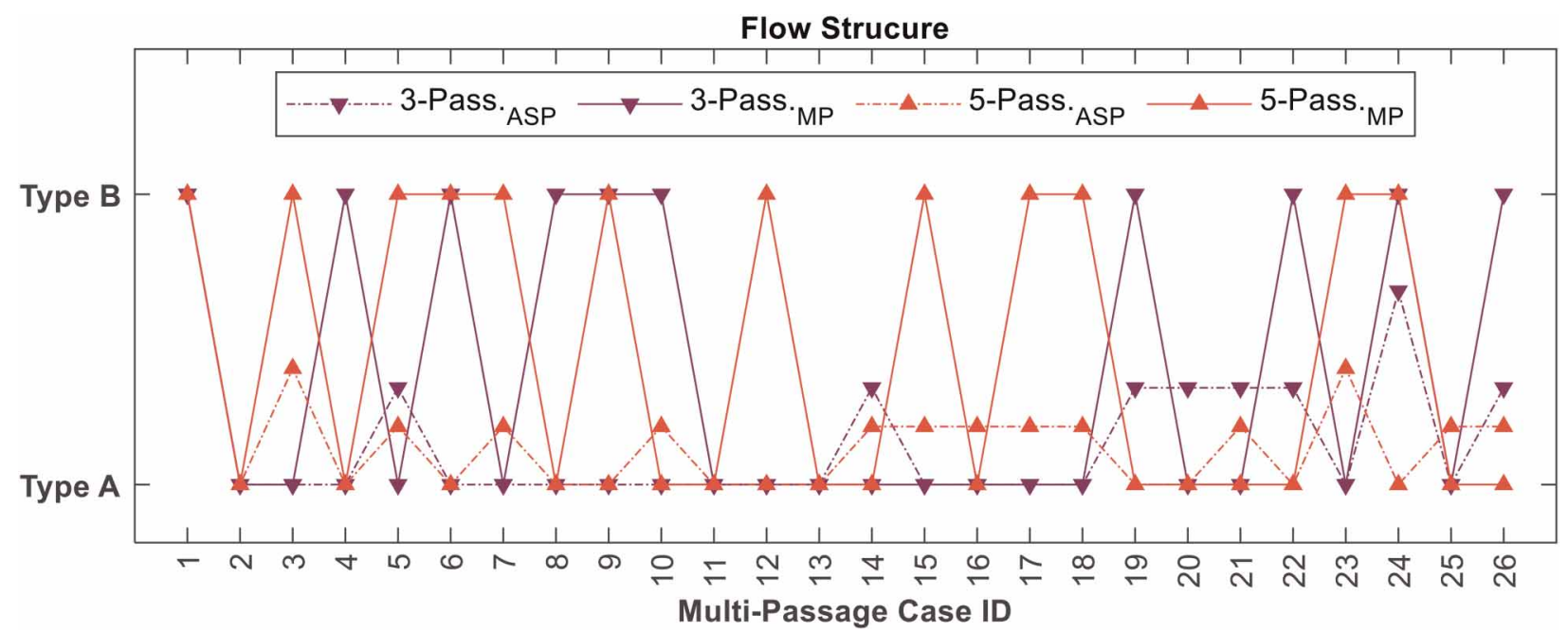

Figure 9. Flow structures observed in the multi-passage (MP) CFD models and the "average" flow structure from the single-passage (ASP) CFD models of the corresponding constituent blades. 
- the mean and standard deviation of the MP and ASP predictions are indicated by a circle marker and error bar respectively along the appropriate axes.

Figure 10a and 10b show good agreement between the ASP and MP results for the mass-averaged $\beta_{\text {exit }}$ and $Q$ as the data points lie along the one-to-one line. The mean mass-averaged $\beta_{\text {exit }}$ and mean $Q$ from the 3-passages and 5 -passages cases are consistent and do not show a noticeable shift when the number of blade passages is increased. The standard deviation, however, reduces with an increasing number of blade passages. The insensitivity of the mass-averaged $\beta_{\text {exit }}$ and $Q$ to the flow structure changes (multi-passage model that exhibit Type B flow are denoted by the subscript "B" and filled-in markers in Figure 10) supports the validity of averaging singlepassage results to predict multi-passage behaviour.

In contrast, Figure 10c shows significant discrepancy between the predicted stage loading $\psi$ from the ASP and MP approaches. For the cases that exhibit Type B flow, the MP values of $\psi$ are typically higher than the ASP

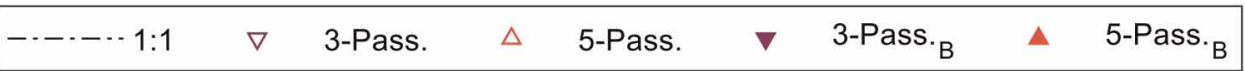

HPT rotor exit flow angle, $\Delta \beta_{\text {exit }}$

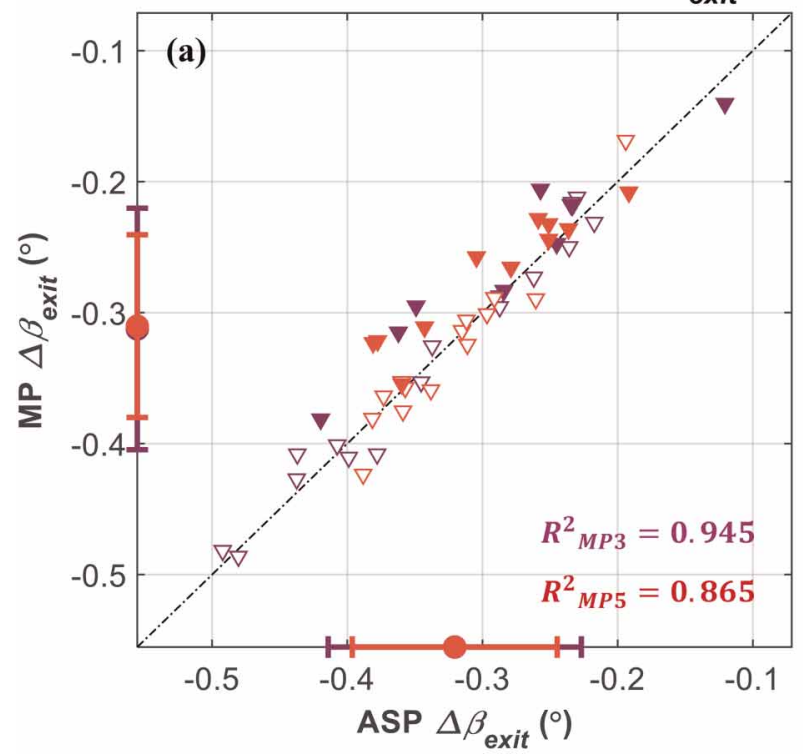

HPT stage loading coeff., $\Delta \psi$

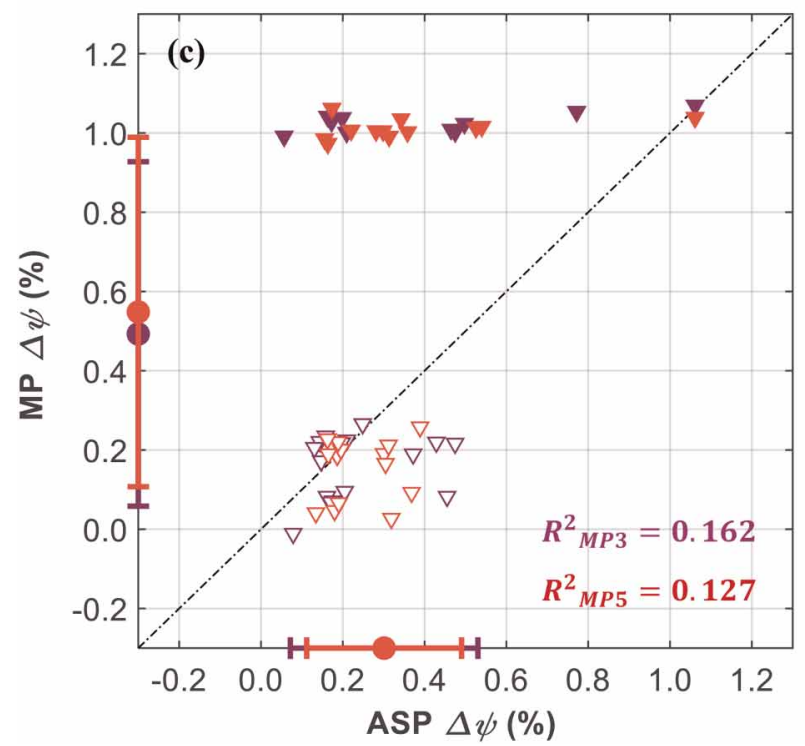

HPT stage capacity, $\Delta Q$

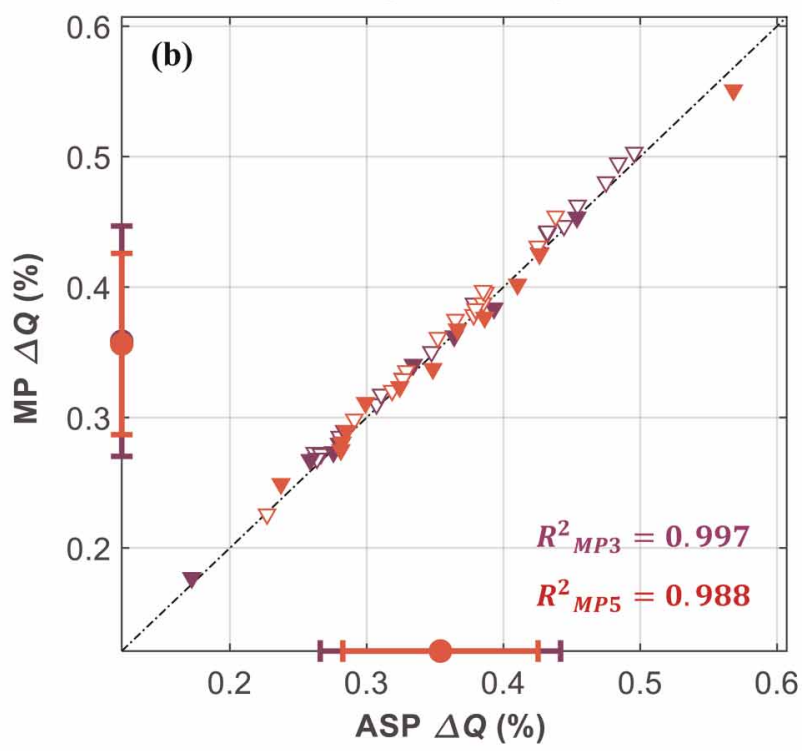

scaled HPT stage efficiency, $\Delta \eta$

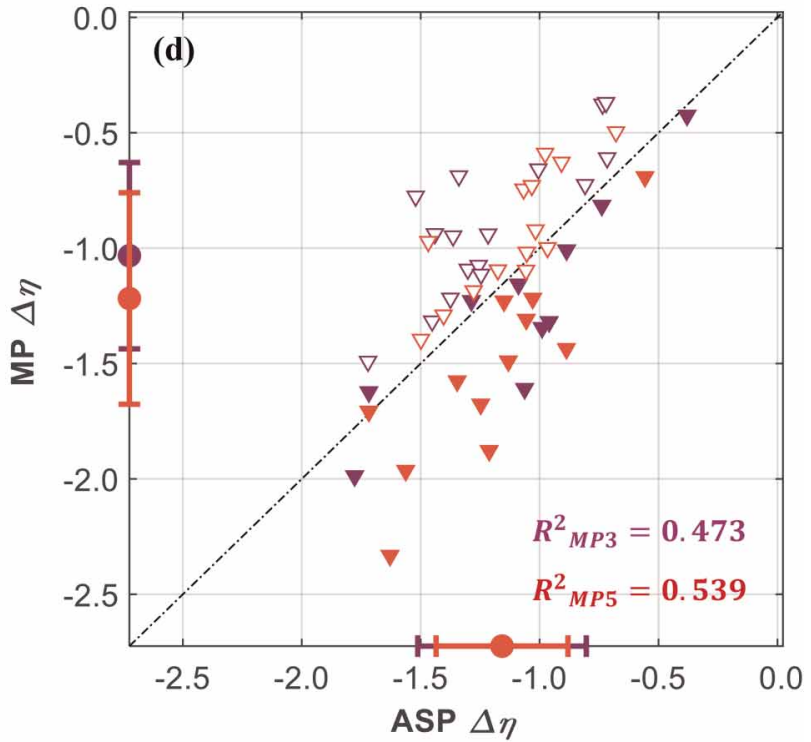

Figure 10. Plots of the averaged single-passage (ASP) values against multi-passage (MP) values from the multipassage CFD models for the: (a) mass-averaged $\beta_{\text {exit }}$ (b) $Q$, (c) $\psi$, and (d) scaled $\eta$. The mean and standard deviation are marked on the appropriate axes with a circle and an error bar respectively. 
values. The exception is the case in the top-right corner of Figure 10c, case index 1 in Figure 9, which was assembled from blades that only exhibited Type B flow. The dependency on the flow structure is also shown by how the cases that exhibit Type B behaviour form a distinct cluster which causes the mean of the MP models to shift (compared to the mean of the ASP values) and increases the standard deviation in the MP results. For efficiency $\eta$, Figure $10 \mathrm{~d}$ shows a weak correlation between the predictions of $\eta$ from the ASP and MP approaches. This shows a significant inter-dependence between the blades and averaging single-passage results is only capable of giving a rough estimate of the multi-passage stage efficiency.

To summarise, the HSV flow structure near the vicinity of the HPT rotor blade tip is bimodal: Type A and Type B flow are observed as two distinct states with no clear intermediate state between the two. The behaviour is sensitive to interaction between adjacent blades and their pressure fields. Considering overall performance parameters, flow angle and capacity are well approximated by averaging the individual single-passage predictions of each blade $\left(R^{2}>0.86\right)$. This approach is significantly less accurate for the stage loading coefficient $\psi$ and efficiency $\eta\left(R^{2}<0.54\right)$.

\section{Part IV: accuracy and computational costs across the hierarchy}

Having examined some of the differences between the predictions obtained from aerodynamic simulation methods of various degrees of realism, attention now returns to the central question motivating this paper, namely what is the trade-off between cost and accuracy of different aerodynamic simulation fidelities?

Figure 11 plots the predicted changes in capacity $Q$ and scaled HPT stage efficiency $\eta$ for two of the 25 blades: blade ID 2 that exhibits Type A flow and blade ID 20 that exhibits Type B flow. The performance changes are plotted on a log-scale for the number of CPU hours that were used to run each simulation. To ensure a valid comparison, the RANS and URANS cases were calculated using only the HPT stage as the computational domain and the same blade (either blade ID 2 or blade ID 20) was used to populate every blade position in the cases where the HPT rotor has more than one blade passage.

On a log-scale, the Euler, RANS, and URANS CFD models occupy three distinct regions. Compared to the predictions from RANS and URANS, Euler models are very quick and take only $\sim 15$ CPU core seconds per blade section. However, the Euler models fall short in the ability to capture the performance changes observed in the higher fidelity methods. The RANS models are all of similar order of magnitude in terms of simulation cost (50-200 CPU core hours) and the URANS models require 5,500 core hours. As the URANS models required the same size sector for the NGV and rotor blade rows, the URANS models had 6 HP NGVs and 11 HPT rotor blades. In addition to these raw computational costs, the complexity in running the models in Figure 11 generally increases with fidelity. Rising computational cost is therefore accompanied by an increase in the time and expertise required to build and manipulate these models.

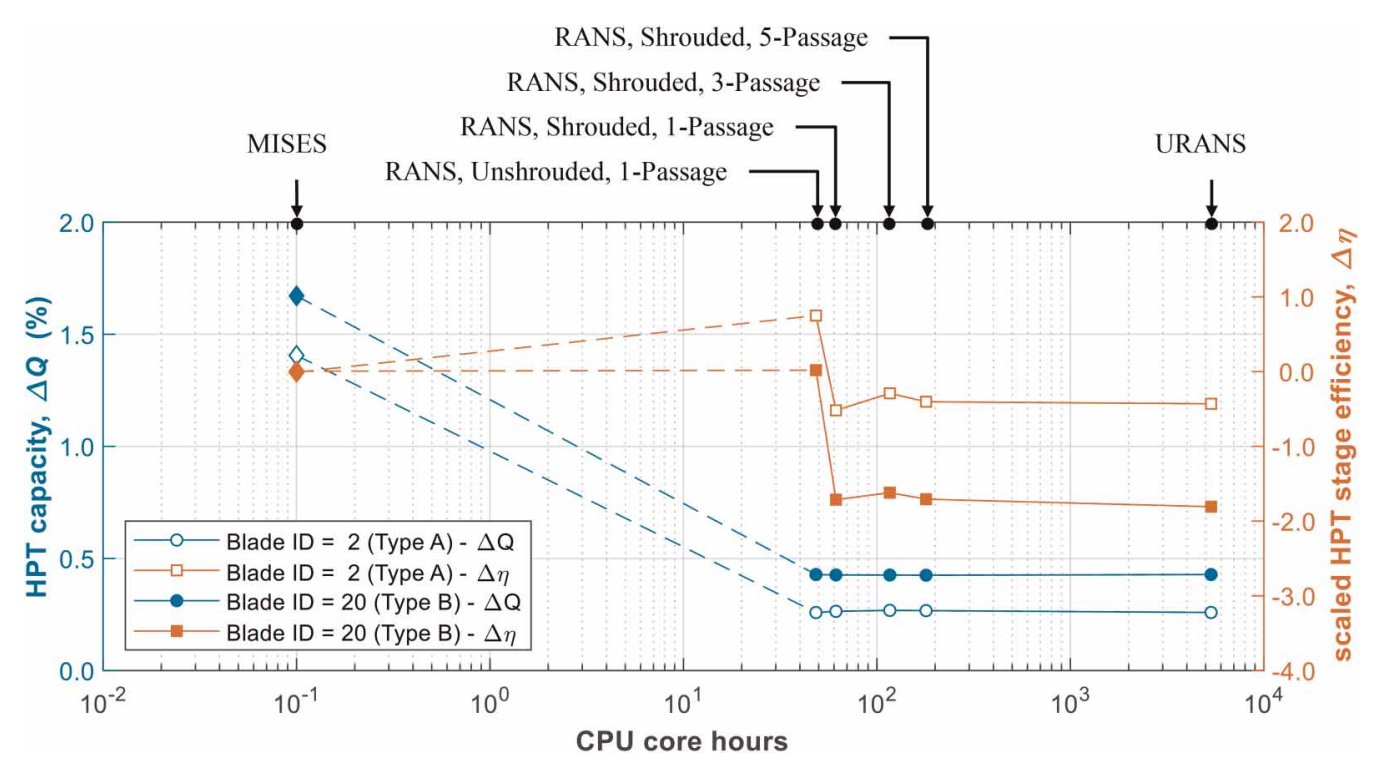

Figure 11. Comparison of the computational costs (CPU core hours) and the predicted performance changes by the various aerodynamic simulations across the aerodynamic simulation hierarchy. 
As a general principle, the required aerodynamic modelling fidelity is driven by the need to capture the aerodynamic phenomena that drive the performance quantities of interest. While it is difficult to draw more specific universal rules for simulation, the following recommendations can be made for Digital Twins of gas turbine blades (in particular, a HPT rotor blade):

1. For predictions of capacity $Q$ and mass-averaged flow angle $\beta_{\text {exit }}$, an unshrouded, single-passage, 3D RANS CFD model is sufficient to predict performance changes: further increases in fidelity do not change the prediction (blue lines in Figure 11). For a 1-stage calculation, this is estimated to take 50 CPU core hours.

2. For the predictions of stage loading $\psi$ and efficiency $\eta$, a shrouded, single-passage, 3D RANS CFD model is recommended as a minimum fidelity. While the predictions do change slightly with further increases in fidelity, the prediction without the shroud is noticeably worse: e.g. the Type B case in Figure 11 shows differences in scaled efficiency of around 2.0 when comparing unshrouded and shrouded cases. For a 1-stage calculation, this is estimated to take $\sim 65 \mathrm{CPU}$ core hours.

3. The approach of using URANS CFD models to support a Digital Twins seems unnecessarily complicated and costly as the predicted performance changes no longer vary significantly beyond a single-passage, shrouded RANS CFD model.

4. As a general design rule, components should have a robustness to variability. The blade tip could be designed to stabilise the HSV structure which would improve the reliability of lower-fidelity models and reduce the required complexity in the aerodynamic simulations.

\section{Conclusions}

Using MISES and Hydra, CFD models of varying degrees of realism were generated and the performance of 25 scrapped HPT rotor blades was assessed. The goal was to determine the required level of fidelity in simulations to obtain physics-based predictions that can be used to support Digital Twin technology for the manufacturing process of gas turbine blades. The performance of the HPT stage was quantified in terms of the rotor exit angle $\beta_{\text {exit, }}$, stage inlet capacity $Q$, stage loading coefficient $\psi$, and stage efficiency $\eta$.

In terms of the bulk flow properties, MISES, although quick, was unable to capture the trend in $\beta_{\text {exit }}$ due to its limitation of only being able to consider quasi-3D blade sections. A shrouded RANS model was the minimum required fidelity to capture a migration of the horseshoe vortex, a fluid mechanic phenomenon that is sensitive to the imposed geometry changes. If the pressure field is weakened sufficiently, the horseshoe vortex could migrate out of the upstream cavity and wrap around the leading edge of the blade. The aerodynamic changes that were introduced by varying HPT rotor blade geometry were observed as far downstream as the IPT rotor blade and this requires a multi-stage computational domain if such interaction effects are of interest.

Multi-passage calculations were performed to assess interactions between neighbouring blades and these interactions affect the casing horseshoe vortex behaviour. In general, flow angle $\beta_{\text {exit }}$ and capacity $Q$ are largely unaffected by the horseshoe vortex behaviour: the multi-passage values are well approximated by a simple average of the constituent single-passage cases. In contrast, stage loading $\psi$ showed a strong dependence on the state of the casing horseshoe vortex and averaging single passage results leads to higher errors. Similarly, efficiency $\eta$ showed a high degree of scatter which suggests additional inter-dependencies between blades that cannot be captured by simply averaging single passage results.

To support Digital Twins of a HPT rotor blades, an unshrouded single-passage 3D RANS model is sufficient to assess flow angle and stage capacity changes, while a shrouded single-passage 3D RANS model is the minimum required fidelity to give an estimate of stage loading and efficiency. The fidelity of the CFD models could be reduced if the casing flow structure change were avoided by designing a blade that stabilises the horseshoe vortex. URANS simulations are far too costly for extensive use, but serve as a useful benchmark for this study.

\section{Nomenclature}

$\begin{array}{ll}\Delta & \text { Difference } \\ \beta_{\text {exit }} & \text { Exit flow angle } \\ \eta & \text { Stage efficiency } \\ \tau & \text { Viscous shear } \\ \psi & \text { Stage loading coefficient } \\ F_{\vartheta} & \text { Tangential component of force } \\ P_{0, \text { inlet }} & \text { Stagnation pressure at stage inlet }\end{array}$


Q Stage inlet capacity

$S \quad$ Surface

$T_{0 \text {,inlet }}$ Stagnation temperature at stage inlet

$U \quad$ Blade velocity

$V_{\vartheta} \quad$ Tangential velocity

$V_{x} \quad$ Axial velocity

$h_{0} \quad$ Total enthalpy

$\dot{m} \quad$ Mass flow

$\dot{m}_{\text {inlet }}$ Mass flow at stage inlet

$p \quad$ Static pressure

$r \quad$ Radius

\section{Abbreviations}

3D Three-Dimensional

ASP Average of Single Passage

CFD Computational Fluid Dynamics

CMM Coordinate Measuring Machine

CPU Central Processing Unit

DNS Direct Numerical Simulation

HP High Pressure

HPT High Pressure Turbine

HSV Horseshoe Vortex

IP Intermediate Pressure

IPT Intermediate Pressure Turbine

LES Large Eddy Simulation

MISES Multiple blade Interacting Streamtube Euler Solver

MP Multi-Passage

NGV Nozzle Guide Vane

PADRAM Parametric Design \& Rapid Meshing

Q3D Quasi-Three-Dimensional

RANS Reynolds-Averaged Navier-Stokes

STL Stereolithography

TE Trailing Edge

URANS Unsteady Reynolds-Averaged Navier-Stokes

\section{Acknowledgements}

The authors would like to acknowledge the contributions of Dr Graham Bing in securing the STL meshes and Mr Lars Högner and his team at TU Dresden for developing and sharing BladeCleaner. The authors would also like to thank the Engineering and Physical Sciences Research Council for funding. Finally, the authors would like to express their gratitude to Rolls-Royce plc for funding, access to PADRAM and Hydra, and for allowing the findings of this paper to be published.

\section{Funding sources}

Rolls-Royce plc, Engineering and Physical Sciences Research Council Impact Acceleration Account.

\section{Competing interests}

Wen Yao Lee declares that he has no conflict of interest. William N. Dawes declares that he has no conflict of interest. John D. Coull declares that he has no conflict of interest.

\section{References}

Clark J. P., Beck J. A., Kaszynski A. A., Still A., and Ni R.-H. (2018). The effect of manufacturing variations on unsteady interaction in a transonic turbine. Journal of Turbomachinery. 140 (6): 061007. https://doi.org/10.1115/1.4039361

Coull J. D. (2017). Endwall loss in turbine cascades. Journal of Turbomachinery. 139 (8): 081004. https://doi.org/10.1115/1.4035663

Dow E. A. and Wang Q. (2015). The implications of tolerance optimization on compressor blade design. Journal of Turbomachinery. 137 (10): 101008. https://doi.org/10.1115/1.4030791 
Drela M. (1985). Two-Dimensional Transonic Aerodynamic Design and Analysis Using the Euler Equations. PhD. Massachusetts Institute of Technology. Available at: http://hdl.handle.net/1721.1/14974

Drela M. and Youngren H. (2008) A User's Guide to MISES 2.63. MIT Aerospace Computational Design Laboratory.

Duffner, J.D. (2008). The Effects of Manufacturing Variability on Turbine Vane Performance. MSc. Massachusetts Institute of Technology. Available at: http://hdl.handle.net/1721.1/57599

Fuller A., Fan Z., Day C., and Barlow C. (2020). Digital twin: enabling technologies, challenges and open research. IEEE Access. 8: 108952-108971. https://ieeexplore.ieee.org/document/9103025/

Garzon V. E. and Darmofal D. L. (2003). Impact of geometric variability on axial compressor performance. Journal of Turbomachinery. 125 (4): 692-703. https://doi.org/10.1115/1.1622715

Goodhand M. N., Miller R. J., and Lung H. W. (2015). The impact of geometric variation on compressor two-dimensional incidence range. Journal of Turbomachinery. 137 (2): 021007. https://doi.org/10.1115/1.4028355

Heinze K., Meyer M., Scharfenstein J., Voigt M., and Vogeler K. (2014). A parametric model for probabilistic analysis of turbine blades considering real geometric effects. CEAS Aeronautical Journal. 5: 41-51. https://doi.org/10.1007/s13272-013-0088-6

Högner L., Voigt M., Vogeler K., Meyer M., and Berridge C. (2015). A Curvature Based Algorithm for Treatment of Cooling Holes in Polygon Meshes of Turbine Blades, Paper No: GT2015-42841. In: ASME Turbo Expo 2015: Turbine Technical Conference and Exposition. Montreal, Quebec, Canada: ASME, Volume 2B: Turbomachinery, V02BT39A025. Available at: https://doi.org/10.1115/ GT2015-42841

Högner L., Nasuf A., Voigt P., Voigt M., Vogeler K., et al. (2016). Analysis of High Pressure Turbine Nozzle Guide Vanes Considering Geometric Variations, Paper No: GT2016-57502. In: ASME Turbo Expo 2016: Turbomachinery Technical Conference and Exposition. Seoul, South Korea: ASME, Volume 2C: Turbomachinery, V02CT45A025. Available at: https://doi.org/10.1115/GT2016-57502

Högner L., Knebel S., Voigt M., Mailach R., and Meyer M. (2017). Quantification of X-Ray Measurement Uncertainty Based on Optical Measurement Data of Turbine Blades, Paper No. GT2017-63704. In: ASME Turbo Expo 2017: Turbomachinery Technical Conference and Exposition. Charlotte, North Carolina, USA: ASME, Volume 2C: Turbomachinery, V02CT47A008. Available at: https://doi.org/10.1115/GT2017-63704

Kamenik J., Voutchkov I., Toal D. J. J., Keane A. J., Högner L., et al. (2018). Robust turbine blade optimization in the face of real geometric variations. Journal of Propulsion and Power. 34 (6): 1479-1493. https://doi.org/10.2514/1.B37091

Kapsis M. and He L. (2018). Analysis of aerothermal characteristics of surface microstructures. Journal of Fluids Engineering. 140 (5): 051104. https://doi.org/10.1115/1.4038667

Lange A., Voigt M., Vogeler K., Schrapp H., Johann E., and Gümmer V. (2012). Impact of manufacturing variability on multistage highpressure compressor performance. Journal of Engineering for Gas Turbines and Power. 134 (11): 112601. https://doi.org/10.1115/1. 4007167

Lee W. Y., Dawes W. N., and Coull J. D. (2019a). Physics-Based Part Orientation and Sentencing: A Solution to Manufacturing Variability, Paper No: GT2019-91591. In: ASME Turbo Expo 2019: Turbomachinery Technical Conference and Exposition. Phoenix, Arizona, USA: ASME, Volume 2D: Turbomachinery, V02DT46A019. Available at: https://doi.org/10.1115/GT2019-91591

Lee W. Y., Dawes W. N., Coull J. D., and Goenaga F. (2019b). The Impact of Manufacturing Variability on Multi-Passage High Pressure Turbine Aerodynamics, Paper No: AIAA 2019-1950. In: AIAA Scitech 2019 Forum. San Diego, California, USA: AIAA. Available at: https://doi.org/10.2514/6.2019-1950

Menter F. R. (1992). Improved Two-Equation k-w Turbulence Models for Aerodynamic Flows. National Aeronautics and Space Administration. Available at: https://ntrs.nasa.gov/archive/nasa/casi.ntrs.nasa.gov/19930013620.pdf

Moinier P. and Giles M. B. (1998). Preconditioned Euler and Navier-Stokes Calculations on Unstructured Meshes. In: 6th ICFD Conference on Numerical Methods for Fluid Dynamics. Oxford, UK.

Rolls-Royce plc. (2009). The HYDRA User's Guide. Rolls-Royce plc.

Shahpar S. and Lapworth L. (2003). PADRAM: Parametric Design and Rapid Meshing System for Turbomachinery Optimisation, Paper No: GT2003-38698. In: ASME Turbo Expo 2003. Atlanta, Georgia, USA: ASME, Volume 6: Turbo Expo 2003, Parts A and B, pp. 579-590. Available at: https://doi.org/10.1115/GT2003-38698

Zamboni G. and Adami P. (2016). On the Unsteady Interaction Between the Leakage and the Main Passage Flow in a High Pressure Turbine Rig: CFD URANS Investigations and Comparison With the Rig Test Data. In: ASME Turbo Expo 2016: Turbomachinery Technical Conference and Exposition. Seoul, South Korea: ASME, Volume 2B: Turbomachinery, V02BT38A002. Available at: https://doi.org/10.1115/GT2016-56041 